Tableau 1

Effets indésirables cardiovasculaires chez les patients traités par carfilzomib ( $\mathbf{~ = ~ 5 6 ) . ~}$

Les données sont données en chiffres (\%).

\begin{tabular}{|c|c|c|c|c|}
\hline & Grade 1-2 & Grade 3-4 & Grade 5 & Total \\
\hline Insuffisance cardiaque & $12(21)$ & $11(20)$ & - & $23(41)$ \\
\hline Hypertension artérielle & - & $13(23)$ & - & $13(23)$ \\
\hline Douleur thoracique & $8(14)$ & $1(2)$ & - & $9(16)$ \\
\hline Hypertension pulmonaire & $2(3,5)$ & $2(3,5)$ & - & $4(7)$ \\
\hline Arythmie & $2(3,5)$ & $2(3,5)$ & - & $4(7)$ \\
\hline Syndrome coronarien aigu (SCA) & $1(2)$ & $1(2)$ & $1(2)$ & $3(6)$ \\
\hline
\end{tabular}

observé de complications thromboemboliques. Un décès, attribué à un infarctus du myocarde, est survenu dans les $24 \mathrm{~h}$ après une perfusion de carfilzomib. Quatre-vingt-six pour cent de ces EICV sont survenus au cours des trois premiers mois de traitement, et $33 \%$ au cours du premier mois.

L'hydratation associée aux perfusions ( $\geq$ ou $<1$ L de sérum physiologique par semaine), la dose de carfilzomib reçue (27 versus $56 \mathrm{mg} / \mathrm{m}^{2}$ ou plus) ou la durée de perfusion (sur $10 \mathrm{~min}$ ou plus longue) ne semblait pas modifier la survenue de ces EICV, tout comme la prise d'IMiD ou la dose de dexaméthasone associée.

En revanche, un taux de peptides natriurétiques de type $\mathrm{B}$ (BNP) $>100 \mathrm{pg} / \mathrm{mL}$ ou de $\mathrm{N}$ terminalpro-BNP (NT-proBNP) $>125 \mathrm{pg} / \mathrm{ml}$ avant traitement était associé à une plus grande incidence d'EICV (odds ratio 10,8, p < 0,001). L'élévation de ces marqueurs par rapport au baseline dans les trois premières semaines de traitement était également un facteur de risque (odds ratio 36 ; $\mathrm{p}<0,001)$. La troponine, l'ECG, et l'échographie cardiaque n'étaient pas prédictifs de la survenue de ces EICV. Pour l'ensemble de la population, le traitement par IP a pu être poursuivi sans modification dans $48 \%$ des cas $(\mathrm{n}=31) ; 41 \%(\mathrm{n}=26)$ des EICV ont imposé un décalage et/ou une réduction des doses; $11 \%$ des patients $(n=7)$ ont dû interrompre le traitement définitivement. La majorité de ces EICV était transitoire, avec une résolution des paramètres biologiques en 24,5 jours en médiane.

En conclusion, les EICV sont fréquents avec le carfilzomib; en plus de l'insuffisance cardiaque et de l'HTA, il faut également signaler la survenue de syndromes coronariens aiguës, d'arythmie, et d'hypertension pulmonaire. Le BNP et le NT-proBNP semblent être des marqueurs fiables, et devraient être intégrés dans la surveillance habituelle de ces patients. Il est intéressant de noter que cette toxicité est indépendante de la dose de carfilzomib reçue. Heureusement, cette toxicité semble résolutive dans la plupart des cas ; les modalités de réintroduction du carfilzomib dans cette situation restent encore à définir. ]

\section{Références}

[1] Li W, Garcia D, Cornell RF, et al. Cardiovascular and Thrombotic Complications of Novel Multiple Myeloma Therapies: A Review. JAMA Oncol 2017 3 : 980-8.

[2] Cornell RF, Ky B, Weiss BM, et al. Prospective Study of Cardiac Events During Proteasome Inhibitor Therapy for Relapsed Multiple Myeloma. J Clin Oncol 2019 ; 37 : 1946-55.

\title{
Traitement par sélinexor du myélome multiple triple réfractaire
}

\section{Nicolas Stocker}

es progrès thérapeutiques récents, tels que le développement des agents immunomodulateurs, des inhibiteurs du protéasome ou des anticorps monoclonaux, ont significativement amélioré la survie des patients atteints de myélome multiple [1]. Cependant, les rechutes restent fréquentes, avec des myélomes multiples pouvant être réfractaires à ces trois classes thérapeutiques (dits myélomes multiples triple réfractaires). Le sélinexor, un inhibiteur sélectif de l'exportine 1 (XP01), une protéine responsable de l'export nucléaire et surexprimée dans ce contexte, pourrait être une option de traitement prometteuse chez ces patients. Les études précliniques ont révélé une activité apoptotique antitumorale par rétention nucléaire et activation de protéines suppressives 
de tumeurs chez des patients atteints de myélome multiple en rechute ou réfractaire [2]. Ces données ont alors conduit à la réalisation de l'essai de phase IIb présenté ici évaluant le sélinexor, combiné à la dexaméthasone, chez des patients atteints de myélomes multiples triple réfractaires [3].

Entre mai 2015 et mars 2018, 123 patients atteints de myélomes multiples triple réfractaires ont été inclus dans cette étude. L'âge médian des patients était de 65 ans (40-86) et le délai médian depuis le diagnostic de six ans (1-23). Cinquantetrois pour cent des patients présentaient des anomalies cytogénétiques dites de haut risque - délétion $\mathrm{du} \quad 17 \mathrm{p}$ (del[17p]), translocation $(4 ; 14)(\mathrm{t}[4 ; 14])$ ou $\mathrm{t}(14 ; 16)$ - tandis que le nombre médian de lignes antérieures de traitement était de sept (3-18). Quatre-vingt-six patients $(70 \%)$ avaient reçu du daratumumab, 102 patients (84\%) une autogreffe de cellules souches hématopoïétiques et deux patients (2\%) un traitement antérieur par cellules $\mathrm{T}$ à récepteurs antigéniques chimériques (CAR-T). Au total, quatre-vingt-trois patients (68\%) présentaient un myélome multiple pentaréfractaire (bortézomib, carfilzomib, lénalidomide, pomalidomide et daratumumab).
Suite à l'initiation de la combinaison sélinexor-dexaméthasone, 118 patients (96\%) ont interrompu le traitement, principalement en raison d'un effet indésirable grave ou de la progression de la pathologie, et la durée médiane de traitement était de neuf semaines (1-60). Les principaux effets indésirables de grade $\geq 3$ étaient hématologiques, incluant des thrombopénies (59\%), des anémies (44\%) et des neutropénies (21\%). Par ailleurs, $22 \%$ des patients présentaient des hyponatrémies de grade $\geq 3$. Ces effets indésirables graves, imputables au sélinexor ou à la dexaméthasone, ont conduit à l'interruption du traitement chez $18 \%$ des patients ; et des modifications de dose, voire des arrêts de traitement, furent nécessaires pour $80 \%$ des patients.

En termes d'efficacité, le taux de réponse globale était de $26 \%$ (95\% CI : 19-35), avec respectivement 20, 5 et $2 \%$ des patients en réponse partielle, en réponse partielle de très bonne qualité et en réponse complète stringente. Le délai médian de réponse était quatre mois (1-14) et la durée médiane de réponse de quatre mois (4-11). Les médianes de survie sans progression et globale étaient respectivement de quatre mois (95\%CI : 3-5) et neuf mois (95\%CI : 6-11). Chez les patients ayant présenté au moins une réponse minimale, la médiane de survie globale atteignait seize mois (95\% CI : 13-NA). Enfin, la quantification des ARNm d'XPO1 et de quatre protéines régulatrices (IRF3, ARL2BP, ZBTB17 et ATRX) ne permettait pas de distinguer les patients répondeurs au traitement.

En conclusion, cette étude de phase IIb démontre l'efficacité et la faisabilité d'un traitement par sélinexordexaméthasone chez les patients atteints de myélomes multiples triple réfractaires. Cette combinaison innovante permet l'obtention d'une réponse chez $26 \%$ de ces patients lourdement prétraités, de haut risque et en majorité pentaréfractaires. Bien que les effets indésirables hématologiques de grade $\geq 3$ soient fréquents, la progression rapide de ces patients et l'absence d'alternative thérapeutique rendent ce profil de toxicité acceptable. ]

\section{Références}

[1] Kumar SK, Dispenzieri A, Lacy MQ, et al. Continued improvement in survival in multiple myeloma: changes in early mortality and outcomes in older patients. Leukemia $2014 ; 28: 1122-8$.

[2] Vogl DT, Dingli D, Cornell RF, et al. Selective Inhibition of Nuclear Export With Oral Selinexor for Treatment of Relapsed or Refractory Multiple Myeloma. J Clin Oncol 2018 ; 36 : 859-66.

[3] Chari A, Vogl DT, Gavriatopoulou M, et al. Oral Selinexor-Dexamethasone for Triple-Class Refractory Multiple Myeloma. N Engl J Med 2019 ; 381 : 727-38.

\section{Toxicité hématologique précoce et tardive des cellules $T$ à récepteurs antigéniques chimériques anti-CD19}

\section{Jérôme Paillassa}

es cellules $\mathrm{T}$ à récepteurs antigéniques chimériques (CAR-T) anti-CD19 ont récemment montré leur remarquable efficacité dans les leucémies aiguës lymphoblastiques $B$ (LAL B), les lymphomes non hodgkiniens B (LNH B) et les leucémies lymphoïdes chroniques (LLC). Les principales toxicités sont le syndrome de relargage cytokinique (SRC), la toxicité neurologique centrale et l'hypogammaglobulinémie liée à l'hypoplasie B. Des neutropénies, thrombopénies et anémies ont également été décrites, dont certaines tardives par rapport à l'injection des CAR-T [1, 2]. Une étude israélienne, récemment publiée dans Bone
Marrow Transplantation, a exploré ces cytopénies précoces et tardives et a tenté d'en expliquer les mécanismes physiopathologiques [3].

Les données de cette étude sont issues d'un essai prospectif de phase Ib/II dans lequel trente-cinq patients (quatorze enfants et vingt et un adultes, dix-neuf LAL et seize LNH, au moins deux lignes de traitements anté- 